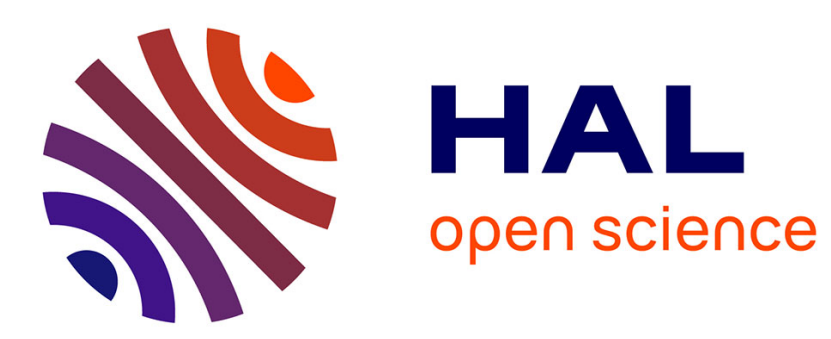

\title{
Progressive entry and the incentives to invest in alternative infrastructures
}

Marc Bourreau, Joeffrey Drouard

\section{To cite this version:}

Marc Bourreau, Joeffrey Drouard. Progressive entry and the incentives to invest in alternative infrastructures. Journal of Regulatory Economics, 2014, 45 (3), pp.329-351. 10.1007/s11149-014-9248-9 . hal-01016366

\section{HAL Id: hal-01016366 https://hal-univ-rennes1.archives-ouvertes.fr/hal-01016366}

Submitted on 30 Jun 2014

HAL is a multi-disciplinary open access archive for the deposit and dissemination of scientific research documents, whether they are published or not. The documents may come from teaching and research institutions in France or abroad, or from public or private research centers.
L'archive ouverte pluridisciplinaire HAL, est destinée au dépôt et à la diffusion de documents scientifiques de niveau recherche, publiés ou non, émanant des établissements d'enseignement et de recherche français ou étrangers, des laboratoires publics ou privés. 


\title{
Progressive Entry and the Incentives to Invest in Alternative
}

\author{
Infrastructures*
}

Marc Bourreau ${ }^{\dagger}$ and Joeffrey Drouard ${ }^{\ddagger}$

February 14, 2014

\begin{abstract}
In this paper we study an entrant's incentives to build a network infrastructure, when there is an initial phase of service-based competition where it leases access to the incumbent's infrastructure. We build a model in which the phase of service-based competition allows the entrant to step into the market by progressively acquiring market experience. We show that the acquisition of experience in the phase of service-based competition delays the entrant's investment when the prospects for infrastructure investment are good, and accelerates investment otherwise. We also show that when the acquisition of experience depends on the entrant's current customer base and facility-based entry is a long-term possibility, setting a low access price can accelerate the entrant's investment.
\end{abstract}

Keywords: Entry; Infrastructure investment; Access pricing; Telecommunications.

JEL Codes: L51; L96.

\footnotetext{
*We thank Axel Gautier, Steffen Hoernig, Pınar Doğan and Wilfried Sand-Zantman for their useful remarks. We also thank audiences at the EARIE Conference (Ljubljana, Slovenia). Finally, we thank the Editor, Michael A. Crew, and two anonymous referees for valuable suggestions. Financial support from Orange is gratefully acknowledged.

${ }^{\dagger}$ Telecom ParisTech and CREST-LEI, Paris, FRANCE. E-mail: marc.bourreau@telecom-paristech.fr.

${ }^{\ddagger}$ University of Rennes 1, CREM, France. E-mail: joeffrey.drouard@univ-rennes1.fr.
} 


\section{Introduction}

In the telecommunications industry, many policy makers view achieving facility-based competition (when new entrants rely on their own infrastructure to compete with the incumbent) as the longterm goal. If competition is to function fully, it is indeed necessary for each operator to control its supply chain to the largest possible extent. Larger benefits in terms of new products and services can moreover be expected under this form of competition than under service-based competition (where new entrants rely on the incumbent's infrastructure to provide services to end consumers). However, as the deployment of new infrastructures involves large sunk costs, potential entrants are rarely able to build their own facilities from the outset. This is why a large majority of national regulatory authorities have mandated incumbents to offer access to their networks to new entrants, so that service-based competition can develop rapidly. ${ }^{1}$

Although service-based competition is clearly beneficial in the short term, its longer-term effects on facility-based competition have generated extensive debates. On the one hand, a traditional view is that there is a conflict between these two forms of competition. Indeed, a phase of service-based competition introduces an opportunity cost of building an alternative infrastructure, and hence lowers the entrants' investment incentives. We refer to this effect as the "replacement effect." On the other hand, others have argued that a phase of service-based competition allows entrants to step into the market and to acquire market experience progressively, which eventually enhances their investment incentives. In other words, service-based competition and facility-based competition are "complements" rather than "substitutes" in promoting competition. We refer to the effect of market experience acquisition on investment incentives as the "stepping stone effect."

An important assumption from the ladder of investment approach ${ }^{2}$ is that the stepping stone effect facilitates investment. That is, given that the replacement effect is neutralized, service-based and facility-based competition are complements. ${ }^{3}$ In this paper we propose a model in which an entrant acquires market experience progressively as it operates in the market. We then study the impact of a phase of service-based competition, and more specifically of the stepping stone effect, on facility-based entry.

\footnotetext{
${ }^{1}$ For example, in the broadband market, unbundling of the local loop (i.e., regulated access to the incumbent's copper local loop) has become mandatory in most industrialized countries. Recently, the European Commission also issued a Recommendation requiring some kind of regulated access to next generation access (fibre) networks (EU, 2010).

${ }^{2}$ See Cave (2006). This approach is often referred to as the stepping stone argument in the US.

${ }^{3}$ As Bourreau et al. (2010) argue, the second assumption behind the ladder of investment approach is that the regulator has the instruments to neutralize the replacement effect.
} 
We consider two firms, an incumbent and a potential entrant. The entrant can enter the market either by acquiring access to the incumbent's facilities (which gives rise to service-based competition) and/or by building its own infrastructure (which gives rise to facility-based competition). The access price to the incumbent's infrastructure is exogenous and set by the regulator. We also assume that the investment cost declines over time and that it is not viable to invest at the outset; therefore, the entrant has to wait before installing its own infrastructure. When it enters the market, on the basis of either services or facilities, the entrant begins to acquire market experience.

In our setting, market experience encompasses different phenomenons. For example, consumers could initially perceive the entrant's service quality as low, but this perceived quality could increase over time as the entrant operates in the market. Prior to entry, consumers might also be unaware of the existence of the entrant. Once the entrant has entered the market, the proportion of informed consumers could gradually increase over time, allowing the entrant to build a customer base.

We begin by studying a baseline case where the acquisition of experience depends only on the time spent in the market. We show that the stepping stone effect affects investment incentives in two opposite ways. On the one hand, as it has accumulated experience during the phase of service-based competition, the entrant anticipates higher profits under facility-based competition, which incentivizes investment. On the other, the entrant may benefit from prolonging the phase of service-based competition to acquire more experience, and gain higher profits under facility-based competition, which tends to delay investment. When facility-based entry is a long-term perspective, the former effect dominates and the stepping stone effect accelerates investment. Otherwise, if facility-based entry is a short-term possibility, the latter effect is the strongest and the stepping stone effect delays the entrant's investment. In other words, taking the replacement effect as given, a process of market experience acquisition does not necessarily make service-based competition and facility-based competition "complements".

We then show that if facility-based entry is possible in the short-term, the phase of servicebased competition delays facility-based entry, compared to a benchmark without access. However, if facility-based entry is a long-term possibility and experience has a strong impact on the entrant's profits, the phase of service-based competition accelerates facility-based entry. In this case only, service-based competition facilitates facility-based entry per se.

In this baseline model, since the acquisition of experience depends only on the time spent in the market, increasing the access price always accelerates investment. When it sets the access price, the regulator therefore faces a standard trade-off between static efficiency and investment incentives. 
We also study the case where the acquisition of market experience depends on the entrant's current customer base, within a specific model of Cournot competition with quality differentiation. If facility-based entry is a short-term possibility, we obtain the same result than in the baseline case: increasing the access price accelerates investment. However, if facility-based entry is a longterm possibility, a low access price accelerates investment, because it allows the entrant to acquire experience rapidly due to a learning-by-doing effect. In this case, the regulator can achieve both static efficiency and early investment via a low access price.

Our paper is related to the literature on access and investment (see Cambini and Jiang (2009) for a recent comprehensive survey). ${ }^{4}$ Bourreau and Doğan (2005 and 2006) analyze the effect of access to an incumbent's infrastructure on an entrant's incentives to build a network. Gans (2001) and (2007), Hori and Mizuno (2006), and Vareda and Hoernig (2010) study the impact of access obligations in investment races between ex-ante symmetric firms. However, these papers do not consider any potential positive impact of the phase of service-based competition on the incentives to enter on the basis of facilities. Therefore, they cannot account for any stepping stone effect.

A few papers consider a possible complementarity between the phases of service-based and facility-based competition. Vareda (2010) studies a two-period model, where the entrant learns about the state of demand once it enters the market. Therefore, service-based competition in the first period resolves the uncertainty about demand, after which the entrant decides whether or not to build its infrastructure. Schutz and Tregouët (2008) build a model that follows the same logic but where the entrant is uncertain about its marginal cost. In these two papers, the resolution of uncertainty can be either good news or bad news for infrastructure investment. In our model, on the other hand, the phase of service-based competition always makes the entrant "stronger," by allowing it to acquire more experience. We show that even in this case, the stepping stone effect can contribute to delaying the entrant's investment.

Avenali et al. (2010) also study a setting where an entrant can acquire experience under servicebased competition. ${ }^{5}$ Experience transforms into a (perceived) high quality only if the entrant invests in its own facilities. Since they also assume that facility-based entry can take place only if the entrant's perceived quality is high, it follows that service-based entry is a necessary prerequisite for facility-based entry. By contrast, in our setting, more experience always transforms into a higher quality, whatever the entrant's current technology (service-based or facility-based). Therefore,

\footnotetext{
${ }^{4}$ See also Valletti (2003) and Guthrie (2006).

${ }^{5}$ Experience acquisition is exogenous in their setting.
} 
service-based entry is not necessary for facility-based entry. However, we show that the existence of a process of experience acquisition is a necessary (but not sufficient) condition for service-based entry to improve the prospects for facility-based entry.

Finally, a number of empirical studies have looked at the relationship between service-based competition and facility-based competition. Most (if not all) of them reject the ladder of investment or stepping stone hypothesis that this relation is positive (i.e., that the stepping stone effect dominates the replacement effect). ${ }^{6}$ Our paper contributes to this literature by characterizing under which conditions the stepping stone effect might be effective in accelerating facility-based entry.

The rest of the paper is organized as follows. In Section 2 we outline the model. In Section 3 we solve for the equilibrium when the acquisition of experience depends only on the time spent in the market. In Section 4 we study the case in which the entrant's acquisition of experience depends also on its current sales. In the last section we conclude.

\section{The Model}

We consider two firms, an incumbent operator (firm $I$ ), who owns a network infrastructure, and a potential entrant (firm $E$ ). The entrant can enter the market either (i) by leasing access to the incumbent's infrastructure at the access price $r \geq 0$ (which leads to "service-based" competition), or (ii) by building its own network infrastructure (which leads to "facility-based" competition). The access price is exogenous and set by the regulator.

Time is continuous, and the horizon is infinite. We denote by $t$ the date at which the entrant enters the market for the first time (on the basis of either services or facilities), and by $T$ its date of facility-based entry. If the entrant does not enter on the basis of services prior to building its infrastructure, the two dates coincide, i.e., $t=T$. Otherwise, we have $t<T$. Finally, at each time $x \geq t$, we denote by $\sigma \equiv x-t$ the time the entrant has spent in the market since it first entered.

Market experience. When it enters the market, the entrant has a low level of market experience, where experience can correspond to the firm's service quality or reputation. It then starts accumulating experience, and at some point of time its level of experience reaches a maximum

\footnotetext{
${ }^{6}$ See, for example, Hazlett and Bazelon (2005) and Hausman and Sidak (2005) for the US, and Crandall and Sidak (2007) and Bacache et al. (2013) for Europe. The only exception is the study by Distaso et al. (2009), who analyze graphically the relation between access prices and the development of alternative broadband infrastructures in Europe, and conclude that national regulatory authorities have adopted policies that are consistent with the ladder of investment approach.
} 
and then remains constant. ${ }^{7}$ Formally, we assume that the entrant's market experience, $\phi$, evolves according to the following process:

$$
\frac{d \phi}{d \sigma}=\left\{\begin{array}{ll}
\alpha+\lambda q_{E} & \text { if } \phi<\bar{\phi} \\
0 & \text { if } \phi \geq \bar{\phi}
\end{array},\right.
$$

where $\alpha>0$ and $\lambda \geq 0$ are parameters, $q_{E}$ is the entrant's current production level, and $\bar{\phi}$ its long-run level of experience. Without loss of generality, we assume that $\phi(0)=0$.

With this formulation, the acquisition of market experience depends on the time the entrant has spent in the market via the parameter $\alpha$, and on the entrant's customer base via the parameter $\lambda$, which generates a "learning-by-doing" effect: the entrant acquires market experience at a faster pace if its current customer base is larger. ${ }^{8}$ The entrant's market experience increases until it reaches the threshold level, $\phi=\bar{\phi}$.

Finally, we assume that the process of market experience acquisition, which is given by (1), is the same under service-based and facility-based competition. However, we will show in Section 4 that when the learning-by-doing effect is operational, in equilibrium experience acquisition goes faster under facility-based competition, because the entrant has a larger customer base under this mode of competition than under service-based competition. ${ }^{9}$

To start with, we study a baseline model in which there is no learning-by-doing effect for the acquisition of experience. This assumption allows us to clearly identify the main effects at play within a highly stylized model, where strategic interactions are limited. It can be relevant, for example, if the word that the entrant is a good-quality provider spreads among all potential consumers, and not only among the entrant's consumers, or if the quality of the entrant is initially lower because it has to make deals with equipment suppliers, content providers, etc., which takes time, but is unrelated to its current customer base. We therefore state the following assumption.

Assumption 1. $\phi$ does not depend on the entrant's customer base, i.e., $\lambda=0$.

Under Assumption 1, since $\phi(0)=0$, the entrant's experience is given by $\phi(\sigma)=\min \{\alpha \sigma, \bar{\phi}\}$.

\footnotetext{
${ }^{7}$ The entrant's long-run level of experience could be equal to the incumbent's level, in which case the entrant competes on a level-playing field with the incumbent (except for the access charge that it has to pay). It could also correspond to a lower or higher level of experience than that of the incumbent.

8 For example, Cave (2006) argues that in the telecommunications industry, new entrants' investments depend on their current revenues and/or their current customer base.

${ }^{9}$ Assuming that the acquisition of experience goes faster under facility-based competition, because for example the entrant controls its own infrastructure, would reinforce this asymmetry.
} 
Since the entrant's experience depends only on the time spent in the market, $\sigma$, no firm can influence the process of market share acquisition through its retail strategy (price, quantity). However, note that $\phi(\sigma)$ is endogenous to the entrant's entry decisions.

In Section 4, we will relax Assumption 1, and consider the (perhaps more realistic) case where the acquisition of market experience depends also on the entrant's current customer base.

Profit flows. At any time, the industry structure is characterized by either monopoly, servicebased competition or facility-based competition. We denote by $\pi_{i}^{j}$ the profit flow of firm $i=I, E$, where $j=M, F, S$ stand for monopoly, facility-based competition and service-based competition, respectively. We assume that the entrant receives a profit flow of 0 when it remains outside the market. The net discounted profit of firm $i=I, E$ is denoted by $\Pi_{i}$, and $\rho>0$ represents the discount factor.

We assume that profit flows are continuous with respect to $\phi$, and we denote by $\Delta \pi_{E}(\phi, r)=$ $\pi_{E}^{F}(\phi)-\pi_{E}^{S}(\phi, r)$ the entrant's profit incentive to switch from service-based to facility-based competition.

Assumption 2. The profit flows are characterized by the following:

(i) For all $r \geq 0$ and $\sigma \geq 0, \partial \pi_{E}^{S}(\phi(\sigma), r) / \partial r \leq 0$.

(ii) For all $r \geq 0$ and $\sigma \geq 0, \pi_{E}^{S}(\phi(\sigma), r) \geq 0$, and $\pi_{E}^{S}(\phi(\sigma), r) \leq \pi_{E}^{F}(\phi(\sigma))$.

(iii) For all $r \geq 0$ and $\phi \geq 0, \partial \pi_{E}^{j}(\phi) / \partial \phi \geq 0$ for $j=F, S$ and $\partial \Delta \pi_{E}(\phi, r) / \partial \phi \geq 0$.

Assumption 2(i) means that a higher access price (weakly) hurts the entrant. This is due to the fact that a higher access price implies a higher perceived marginal cost for the entrant. Assumption 2(ii) implies that the entrant prefers service-based competition to staying out of the market, and that it prefers facility-based competition to service-based competition. Indeed, its perceived marginal cost is higher under service-based competition than under facility-based competition, due to the access price. Moreover, possibilities of differentiation might be greater under facility-based competition, or the entrant might install a more efficient technology when building its own infrastructure. ${ }^{10}$ Assumption 2(iii) states that the entrant's profit and its incentive to switch from

\footnotetext{
${ }^{10}$ Note that if the entrant always prefers service-based competition to facility-based competition, facility-based entry never occurs. Since we are interested in the date of facility-based entry, we do not consider this possibility.
} 
service-based to facility-based competition increase with market experience. ${ }^{11}$

Finally, we assume that the entrant incurs no fixed cost under service-based competition, ${ }^{12}$ and we normalize the marginal cost of access to zero. In Section 4 we provide an example of a competitive setting that satisfies Assumptions 2(i)-(iii).

Investment cost. The cost of building a new infrastructure at date $T$, discounted at time 0 , is denoted by $C(T)$. We make the following assumptions on $C(\cdot)$ :

\section{Assumption 3.}

(i) $C^{\prime}(T) \leq 0, C^{\prime \prime}(T) \geq 0$, and $\lim _{T \rightarrow \infty} C(T)=\underline{C} \geq 0$.

(ii) $C(0)$ is sufficiently large so that facility-based entry never happens at $T=0$.

(iii) $\underline{C}$ is sufficiently small so that the entrant always ends up building its infrastructure.

The cost of building a network infrastructure declines over time due to technological progress. Since we are interested in studying how the entrant's decision to build an infrastructure is affected by the terms of service-based competition and by market experience, we assume away immediate facility-based entry with Assumption 3(ii), and we ignore the case where facility-based entry never occurs with Assumption 3(iii). ${ }^{13}$ Finally, we assume that $C(\cdot)$ is sufficiently convex so that the second-order conditions of profit maximization with respect to the investment date hold.

Timing of the game. The timing of the game is as follows: given $r \geq 0$, the access price set by the regulator, at each moment of time the entrant decides whether to stay out of the market, to lease access to the incumbent's infrastructure, or to build its own infrastructure.

With this timing, we consider that the regulator is able to commit to an access price, ${ }^{14}$ which is

\footnotetext{
${ }^{11}$ Assumption 2(iii) implies that the entrant's incentive to switch from service-based to facility-based competition is higher in the long run than at the date of entry.

${ }^{12}$ In reality, entrants might incur positive entry costs. For example, in the telecommunications industry, with local loop unbundling, there are fixed costs due to co-location and order handling. However, these costs are negligible when compared to the cost of building a new infrastructure.

${ }^{13}$ For larger values of $\underline{C}$, there might be equilibria where the entrant never rolls out its infrastructure. However, our focus is on how the timing of facility-based entry is affected by a phase of service-based competition and a process of market experience acquisition. We therefore assume that facility-based entry always occurs in equilibrium. Note also that in our setting an extremely late date of facility-based entry is equivalent to no facility-based entry.

${ }^{14}$ In our setting there is no commitment problem per se. Once the entrant has invested, there is no point for the regulator to renege on its commitment by setting a low access charge, because the entrant will always be better off using its own infrastructure. This is due to the fact that investment is zero/one in our setting and that there is only one entrant. As Avenali et al. (2010) show, a commitment issue would arise if we were considering a second entrant.
} 
linear and constant over time. ${ }^{15}$ Since our focus is how the acquisition of market experience affects the timing of the entrant's investment, we also ignore a possible reaction from the incumbent to the entry threat. ${ }^{16}$

\section{The Equilibrium}

In this section we first determine the equilibrium date of facility-based entry in a benchmark situation without access to the incumbent's infrastructure. We refer to this benchmark case with the superscript "na" (no access). Then, we study the entrant's entry strategy when it leases access to the incumbent's infrastructure, and refer to this case with the superscript " $a$ " (access). Finally, we compare the investment date with access and without access, and briefly discuss the regulator's choice of the access price.

\subsection{A benchmark: no access}

To analyze the effect of access on the entrant's investment strategy, we start by studying a benchmark where access is not a possibility. ${ }^{17}$ Therefore, the entrant can enter only by building its own infrastructure. It then chooses its investment date, $T$, to maximize its discounted profit,

$$
\Pi_{E}^{n a}(T)=\int_{T}^{\infty} e^{-\rho x} \pi_{E}^{F}(\phi(x-T)) d x-C(T)
$$

Note that at some moment of time, the entrant has reached its maximum level of experience, and does not accumulate experience anymore (i.e., $\phi=\bar{\phi}$ ).

Assuming that $\Pi_{E}^{n a}(T)$ is concave in $T,{ }^{18}$ the optimal investment date, $T^{n a}$, satisfies the following first-order condition,

$$
-\pi_{E}^{F}(\phi(0)) e^{-\rho T^{n a}}+\int_{T^{n a}}^{\infty} e^{-\rho x} \frac{d \pi_{E}^{F}\left(\phi\left(x-T^{n a}\right)\right)}{d T} d x=C^{\prime}\left(T^{n a}\right)
$$

Equation (3) can be interpreted as follows. The entrant equates its marginal discounted facility-

\footnotetext{
${ }^{15}$ We abstract from the optimal regulation of the access price, which would involve setting a more sophisticated access scheme, e.g. a time-dependent access price, as studied by Bourreau and Doğan (2006) or in our working paper version, Bourreau and Drouard (2010).

${ }^{16}$ Bourreau and Doğan (2005 and 2006) study the incumbent's reaction to the entry threat when it can set the access price to its infrastructure. See also Section 4 where we discuss to which extent the incumbent could influence the entrant's acquisition of experience.

${ }^{17}$ For example, the regulator may have banned access.

${ }^{18}$ This is true if the investment cost is sufficiently convex. See Appendix A.
} 
based revenues with its marginal investment cost. At a given date $T$, if its marginal investment cost is higher than its marginal revenue, its optimal decision is to wait. If the entrant waits for a period $d T$, since the investment cost decreases over time, it reduces its investment cost by an amount $C^{\prime}(T) d T$, but it also forgoes some revenues. These foregone revenues are composed of two parts. First, the entrant gives up some facility-based profits during the period $d T$; this corresponds to the term $\pi_{E}^{F}(\phi(0)) e^{-\rho T} d T$ in Equation (3). Second, the entrant does not accumulate market experience during the period $d T$, which decreases its future facility-based discounted profits; this corresponds to the second term, $\left(\int_{T}^{\infty} e^{-\rho x} \frac{d \pi_{E}^{F}(\phi(x-T))}{d T} d x\right) d T$. Note that this term disappears if $\phi$ is constant, that is, if there is no progressive acquisition of market experience for the entrant.

Finally, since we have $C^{\prime \prime} \geq 0$ under Assumption 3(i), the optimal investment date $T^{n a}$ is lower for a higher $\pi_{E}^{F}$; a higher profit flow from facility-based competition implies an earlier date of facility-based entry. Moreover, as $\pi_{E}^{F}$ increases with $\phi$ from Assumption 2(iii), $T^{n a}$ is lower for a higher $\phi^{\prime}$ (i.e., a higher $\alpha$ ). That is, if the entrant acquires market experience more rapidly, it will invest earlier.

Replacing for $\sigma=x-T$ in (2), the first-order condition (3) can be rewritten as

$$
-\rho e^{-\rho T^{n a}} \int_{0}^{\infty} e^{-\rho \sigma} \pi_{E}^{F}(\phi(\sigma)) d \sigma-C^{\prime}\left(T^{n a}\right)=0
$$

From Equation (4), we see that the fact that the entrant acquires market experience progressively delays facility-based competition, because it lowers discounted profits. One natural idea is then to provide some "transitory entry assistance" to new entrants by introducing an initial phase of service-based competition. We study the impact of such an entry-assisting policy in the next subsection.

\subsection{Investment decision with a phase of service-based competition}

When access is a possibility, at the beginning of time, a dominant strategy for the entrant is to request access to the incumbent's infrastructure, as it earns positive profits under service-based competition under Assumption 2(ii), and immediate facility-based entry is not viable under Assumption 3(ii). Therefore, the entrant enters the market on the basis of services at $t=0 .{ }^{19}$ Provided

\footnotetext{
${ }^{19}$ The process of experience acquisition has therefore no influence on the date of service-based entry. This result hinges in particular on our assumption that there is no fixed cost for service-based entry.
} 
that it invests at date $T$ in its own infrastructure, the entrant's discounted profit is

$$
\Pi_{E}^{a}(T)=\int_{0}^{T} e^{-\rho x} \pi_{E}^{S}(\phi(x), r) d x+\int_{T}^{\infty} e^{-\rho x} \pi_{E}^{F}(\phi(x)) d x-C(T)
$$

From date 0 to date $T$, the entrant competes on the basis of services with the incumbent and accumulates market experience. From date $T$ on, the entrant competes on the basis of facilities and continues accumulating experience (until it reaches the threshold level of experience).

We assume that $\Pi_{E}^{a}(T)$ is concave in $T .{ }^{20}$ The optimal facility-based entry date, $T^{a}$, then satisfies the following first-order condition,

$$
-\left[\pi_{E}^{F}\left(\phi\left(T^{a}\right)\right)-\pi_{E}^{S}\left(\phi\left(T^{a}\right), r\right)\right] e^{-\rho T^{a}}-C^{\prime}\left(T^{a}\right)=0 .
$$

The first-order condition (5) shows that the higher the service-based profit flow, $\pi_{E}^{S}$, the later the facility-based entry date, $T^{a}$. This is because the service-based profit flow represents an opportunity cost of facility-based entry for the entrant. This corresponds to a "replacement effect," very similar to the one considered in the licensing literature. ${ }^{21}$

Since $\Delta \pi_{E}(\phi, r)$ increases with $\phi$ under Assumption 2(iii), the higher $\phi^{\prime}$ (i.e., the higher $\alpha$ ), the earlier the investment date, $T^{a}$. In other words, if the entrant acquires market experience faster, facility-based entry takes place earlier.

\subsection{The impact of service-based competition on facility-based entry}

To compare the dates of facility-based entry with and without a phase of service-based competition, we compare the first-order conditions (4) and (5). The first-order condition with a phase of servicebased competition is given by (5), and it can be rewritten as $F O C^{a}\left(T^{a}\right)=0$, where $F O C^{a}(T)=$ $-\left[\pi_{E}^{F}(\phi(T))-\pi_{E}^{S}(\phi(T), r)\right] e^{-\rho T}-C^{\prime}(T)$. Similarly, the first-order condition in the benchmark, which is given by (4), can be written as $F O C^{n a}\left(T^{n a}\right)=0$. To compare the dates of facility-based

\footnotetext{
${ }^{20}$ This is true if the investment cost is sufficiently convex. See Appendix B.

${ }^{21}$ See, for example, Gallini (1984); the incumbent firms reduce the entrants' incentives to innovate by licensing their technologies. In the literature on access and investment, the existence of a replacement effect has already been pointed out by many studies (e.g., see Hori and Mizuno (2006), Vareda and Hoernig (2010), and Bourreau and Doğan $(2005,2006))$.
} 
entry with and without access, using (4) and (5), we rewrite $F O C^{a}$ as follows:

$$
F O C^{a}(T)=F O C^{n a}(T)+\underbrace{\pi_{E}^{S}(\phi(T), r) e^{-\rho T}}_{(\mathrm{I})}+\underbrace{\sum(T)}_{(\mathrm{II})},
$$

where

$$
\Sigma(T)=\rho e^{-\rho T} \int_{0}^{\infty} e^{-\rho x}\left(\pi_{E}^{F}(\phi(x))-\pi_{E}^{F}(\phi(T))\right) d x .
$$

Given that $\Pi_{E}^{a}(T)$ and $\Pi_{E}^{n a}(T)$ are concave, the date of facility-based entry is later with a phase of service-based entry than without such a phase if and only if $F O C^{a}\left(T^{n a}\right)>F O C^{n a}\left(T^{n a}\right)=0$.

Whether $F O C^{a}\left(T^{n a}\right)>0$ or $F O C^{a}\left(T^{n a}\right)<0$ depends on the sign of the sum of term (I) and term (II) in Equation (6), for $T=T^{n a}$. Term (I) represents the replacement effect, that we have discussed earlier. It is positive for all $T$, and therefore it tends to delay facility-based entry. The second term, $\Sigma(T)$, represents the effect of the entrant's acquisition of experience during the phase of service-based competition on its incentives to invest in a network infrastructure. Indeed, if the entrant's experience is immediately at its maximum when it enters the market, this effect is absent, as we have $\Sigma(T)=0$, for all $T$, if $\phi$ is constant. We therefore refer to this effect as the "stepping stone effect." The stepping stone effect accelerates facility-based entry if $\Sigma(T)$ is negative, and delays facility-based entry otherwise. ${ }^{22}$

Intuitively, one could expect that since the stepping stone effect makes the entrant "stronger," it tends to accelerate investment. However, with the following Proposition, we show that a progressive accumulation of experience under service-based competition can in some cases delay the entrant's investment, compared to the benchmark.

Proposition 1 If the date of facility-based entry without access is sufficiently low, the stepping stone effect delays facility-based entry, relative to the benchmark. Otherwise, it accelerates facilitybased entry.

Proof. The sign of $\Sigma(T)$ is the same as the sign of $A(T)$, where

$$
A(T) \equiv \int_{0}^{T} e^{-\rho x}\left(\pi_{E}^{F}(\phi(x))-\pi_{E}^{F}(\phi(T))\right) d x+\int_{T}^{\infty} e^{-\rho x}\left(\pi_{E}^{F}(\phi(x))-\pi_{E}^{F}(\phi(T))\right) d x .
$$

\footnotetext{
${ }^{22}$ Note that the process of experience acquisition also affects investment incentives through current service-based competition profits, but that it is incorporated in the replacement effect, which depends on the current level of experience, $\phi(T)$.
} 
Since $\pi_{E}^{F}(\phi)$ increases with $\phi$ under Assumption 2(iii) and $\phi^{\prime}>0$, we have

$$
\frac{d A(T)}{d T}=-\frac{\phi^{\prime}(T)}{\rho} \frac{\partial \pi_{E}^{F}}{\partial \phi}(\phi(T)) \leq 0
$$

In addition, we have $\lim _{T \rightarrow 0} A(T)>0$ and $\lim _{T \rightarrow \infty} A(T)<0$. Therefore, $A(T)$ is positive for low values of $T$, and negative otherwise. Since the sign of $\Sigma(T)$ is the same as the sign of $A(T)$, it follows that there is a threshold $\widetilde{T}$ such that $\Sigma(T) \geq 0$ for all $T \leq \widetilde{T}$, and $\Sigma(T) \leq 0$ otherwise.

This result highlights that the stepping stone effect impacts facility-based entry in two opposite ways. On the one hand, as it has acquired experience during the phase of service-based competition, the entrant expects higher profits under facility-based competition. On the other, the entrant may benefit from prolonging the phase of service-based competition to acquire more experience, and thereby gain higher profits in the future under facility-based competition.

The first effect gives an incentive to invest earlier than in the benchmark, whereas the second effect gives an incentive to invest later. When the date of facility-based entry is likely to be early, the magnitude of the first effect is very small, as the entrant has not yet acquired much experience. Hence, the second effect dominates and the stepping stone effect delays facility-based entry. On the other hand, if the date of facility-based entry is likely to be late, the magnitude of the first effect is high and it dominates the second effect. The stepping stone then accelerates facility-based entry.

To identify these two opposite effects formally, we rewrite $\Sigma(T)$ as follows,

$$
\Sigma(T)=\left[\pi_{E}^{F}(\phi(0))-\pi_{E}^{F}(\phi(T))\right] e^{-\rho T}-\int_{T}^{\infty} e^{-\rho x} \frac{d}{d T} \pi_{E}^{F}(\phi(x-T)) d x
$$

Now, consider that the entrant decides to delay its investment from date $T$ to date $T+d T$. The stepping stone effect is affected as follows. First, the entrant will not capture the gains associated with the stepping stone effect during the period $d T$. Compared to the no-access benchmark, the entrant will forgo some additional profit, $\left[\pi_{E}^{F}(\phi(0))-\pi_{E}^{F}(\phi(T))\right] e^{-\rho T} d T$, which corresponds to the first term in (7) and to our first positive effect. Second, during this period $d T$, the entrant is going to acquire more experience. Therefore, after the period, the gains associated with a higher level of market experience will be greater for the rest of time. This corresponds to the second term in (7) and to the second negative effect.

If the stepping stone effect delays the entrant's investment, both the stepping stone effect and the replacement effect tend to delay facility-based entry. In this case, service-based competition retards facility-based competition. However, if the stepping stone effect tends to accelerate investment, a 
relevant question is whether a phase of service-based competition can accelerate facility-based entry per se, that is, without a regulation aimed at neutralizing the replacement effect. We have the following result.

Proposition 2 If the date of facility-based entry without access is sufficiently early, a phase of service-based competition delays facility-based entry. If the date of facility-based entry without access is late, a necessary condition for the phase of service-based competition to accelerate investment is that

$$
\pi_{E}^{F}(\bar{\phi})-\pi_{E}^{F}(\phi(0))>\pi_{E}^{S}(\bar{\phi}, r)
$$

Proof. Equation (6) can be rewritten as

$$
F O C^{a}(T)=F O C^{n a}(T)+e^{-\rho T}\left[\pi_{E}^{S}(\phi(T), r)-\pi_{E}^{F}(\phi(T))+\rho \int_{0}^{\infty} e^{-\rho x}\left(\pi_{E}^{F}(\phi(x))\right) d x\right]
$$

This equation shows that the effect of a phase of service-based competition on the date of facilitybased entry depends on the sign of $B\left(T^{n a}\right)$, where

$$
B(T) \equiv \pi_{E}^{S}(\phi(T), r)-\pi_{E}^{F}(\phi(T))+\rho \int_{0}^{\infty} e^{-\rho x}\left(\pi_{E}^{F}(\phi(x))\right) d x
$$

From Assumption 2(iii), $B(T)$ decreases with $T$. Since $\phi^{\prime}>0$ and $\pi_{E}^{S} \geq 0$ from Assumption 2(ii), we also have

$$
\lim _{T \rightarrow 0} B(T)=\rho \int_{0}^{\infty} e^{-\rho x}\left[\pi_{E}^{F}(\phi(x))-\pi_{E}^{F}(\phi(0))\right] d x+\pi_{E}^{S}(\phi(0), r)>0,
$$

which proves the first statement of the Proposition.

As $B(T)$ decreases with $T$, a phase of service-based competition is more likely to accelerate investment if $T$ is high. At the extreme, when $T \rightarrow \infty$, we have

$$
\lim _{T \rightarrow \infty} B(T)=\rho \int_{0}^{\infty} e^{-\rho x} K(x) d x
$$

where $K(x) \equiv \pi_{E}^{F}(\phi(x))-\Delta \pi_{E}(\bar{\phi}, r)$. From Assumption 2(iii) and since $\phi^{\prime}>0$, we have $\partial K / \partial x \geq$ 0 . Since $\lim _{x \rightarrow \infty} K(x)>0, K(x)$ is positive for high values of $x$. Therefore, for $\lim _{T \rightarrow \infty} B(T)<$ 0 to be true, $K(x)$ must be negative for some values of $x$, and in particular for $x=0$. In sum, a necessary condition for service-based competition to accelerate facility-based entry is that $\pi_{E}^{F}(\phi(0))<\Delta \pi_{E}(\bar{\phi}, r)$, that is, $\pi_{E}^{F}(\bar{\phi})-\pi_{E}^{F}(\phi(0))>\pi_{E}^{S}(\bar{\phi}, r)$. 
When there is no experience acquisition (i.e., $\phi$ is constant), service-based competition always delays facility-based entry. Experience acquisition is therefore a necessary condition for servicebased competition to improve the prospects of facility-based entry. Proposition 2 however shows that when the date of facility-based entry without access is early, the phase of service-based competition retards investment, even though the entrant can acquire experience during this phase. If the prospects of facility-based entry are very weak, the phase of access-based competition can in some specific cases accelerate the entrant's infrastructure development. This is true only if Condition (8) holds. This condition states that the gains from the acquisition of experience, in terms of higher facility-based profits, should be sufficiently high, compared to the opportunity cost of facility-based entry (i.e., lost profits from service-based competition).

To summarize, service-based competition is not likely to facilitate facility-based entry per se, except in specific cases where investment is a long-term prospect and experience acquisition has a very strong impact on the entrant's facility-based profits.

\subsection{The impact of the access price on investment and welfare}

Finally, we briefly discuss the regulator's choice of the access price, $r$. The regulator maximizes the discounted social welfare, which is defined as the sum of discounted consumer surplus and industry profits. Since $\phi$ does not depend on $r$, the regulator cannot affect the entrant's acquisition of experience through the access price (e.g., by setting a low access price). However, as the magnitude of the replacement effect decreases with $r$, the regulator can accelerate facility-based entry by increasing the access price.

The regulator therefore faces the standard trade-off between setting a low access price to maximize welfare flows under service-based competition, and setting a high access price to accelerate facility-based entry. As shown by Avenali et al. (2010) and Bourreau and Doğan (2006), this trade off can be alleviated by setting an access price that increases over time. ${ }^{23}$

\section{Market Experience and Customer Base}

So far, we have assumed that market experience depended only on the time spent in the market (i.e., $\lambda=0$ in Equation (1)). In this section we study the case where the acquisition of experience

\footnotetext{
${ }^{23}$ See also our working paper version, Bourreau and Drouard (2010), where we discuss the optimal time-dependent access price in the present setting.
} 
depends also on the entrant's customer base (i.e., $\lambda>0$ ). We adopt the competitive setting of quantity competition with quality differentiation from Katz and Shapiro (1985), ${ }^{24}$ and interpret the entrant's experience as its level of quality. Our main result in that in contrast to the baseline model (see Section 3.4), decreasing the access price can accelerate facility-based entry if facilitybased entry is only a long-term possibility.

In what follows, we begin by presenting the competitive setting. Then, we study the entrant's investment decision. Finally, we study the relation between the access price and the date of facilitybased entry.

\subsection{The competitive setting}

We consider a unit mass of consumers, who decide at each moment of time whether to subscribe to firm $I$ or to firm $E$, or not to subscribe to any of these firms. ${ }^{25}$ Consumers have no switching cost, and therefore their choice of a supplier at a given period of time does not affect their choices at subsequent periods. ${ }^{26}$

The indirect utility of a consumer of type $\tau$ is $U=\tau+\phi_{i}-p_{i}$, where $\phi_{i}$ and $p_{i}$ denote the quality and price of firm $i$, respectively, with $i=I, E$. As we will assume below that firms maximize their current profits when setting quantities, we drop all time index. Consumers' types are uniformly distributed over $[0,1]$. We consider that the entrant's quality corresponds to its market experience (i.e., $\phi_{E}=\phi$ ), and that it evolves according to the process given by Equation (1), with $\lambda>0$. We assume that $\phi_{I}<1$, that $r \in\left[0,\left(1-\phi_{I}\right) / 2\right]$ and that $\bar{\phi}<1+2 \phi_{I}$. The upper bounds for $\phi_{I}$ and $r$ imply that firm $E$ has a positive demand in equilibrium, even if its quality is equal to zero. The upper bound for $\bar{\phi}$ ensures that firm $I$ is active in equilibrium under facility-based competition. Finally, firms have constant marginal production costs that we normalize to zero.

If firm $I$ and firm $E$ are both active in the market, their quality-adjusted prices are necessarily the same, that is, we have $p_{I}-\phi_{I}=p_{E}-\phi_{E}=\widetilde{p}$. The marginal consumer is defined by $\tau=\widetilde{p}$,

\footnotetext{
${ }^{24}$ The assumption of quantity competition seems reasonable to describe competition in telecommunications markets, where firms face capacity constraints. It has also been adopted by other authors, such as Foros (2004) and Nitsche and Wiethaus (2011).

${ }^{25}$ Since consumers make repeat purchases/subscriptions, there is always the same mass of potential consumers at each moment of time. This would not be true with the adoption of a durable good. The consumers who would purchase the durable good at a given period of time would exit the market, reducing the mass of potential adopters in subsequent periods. Additional effects would then arise, affecting the consumers' decision of an adoption time. For example, to the extent that the quality of the good increases with the entrant's experience, a consumer would have an incentive to wait for the entrant to become more experienced to purchase a higher quality durable good. We thank a referee for suggesting us this idea of future research.

${ }^{26}$ Consumers are also too small to influence the market outcome (e.g., the accumulation of experience).
} 
hence, from the uniform distribution assumption, the total demand is $Q=q_{I}+q_{E}=1-\widetilde{p}$. Firm $i=I, E$ then faces the inverse demand function $p_{i}=1-\left(q_{I}+q_{E}\right)+\phi_{i}$.

Firms compete in quantities, and to simplify the analysis, we assume that they maximize their current profit flows, i.e., they are myopic. If firms were not myopic, the entrant would internalize the process of experience acquisition and set a higher quantity. However, the incumbent would not be able to influence the entrant's learning-by-doing process, because when it sets the quantity that maximizes its profit, the incumbent takes the entrant's quantity as given. ${ }^{27}$

This is a consequence of our assumption of quantity competition, and it would be different under price competition. More generally, the incumbent might use some strategy to influence the entrant's experience acquisition (e.g., by investing in quality). ${ }^{28}$ We ignore such strategies here.

Solving for the (short-run) Nash equilibrium, we obtain the profit flows under service-based and facility-based competition. Under service-based competition, the equilibrium profit flows are

$$
\pi_{I}^{S}(\phi, r)=\frac{\left(1+2 \phi_{I}-\phi\right)^{2}}{9}+\frac{\left(5-5 r+\phi_{I}+4 \phi\right)}{9} r, \text { and } \pi_{E}^{S}(\phi, r)=\frac{\left(1+2 \phi-\phi_{I}-2 r\right)^{2}}{9}
$$

Under facility-based competition, the equilibrium profit flows are

$$
\pi_{I}^{F}(\phi)=\frac{\left(1+2 \phi_{I}-\phi\right)^{2}}{9}, \text { and } \pi_{E}^{F}(\phi)=\frac{\left(1+2 \phi-\phi_{I}\right)^{2}}{9}
$$

It can easily be checked that Assumptions 2(i)-(iii) are satisfied.

In the equilibrium of the quantity setting subgame, we have $q_{E}=\left(1+2 \phi-\phi_{I}-2 r\right) / 3$ under service-based competition, and $q_{E}=\left(1+2 \phi-\phi_{I}\right) / 3$ under facility-based competition. The solution of the differential equation (1) is then as follows. If access is not a possibility, since $\phi(0)=0$, the entrant's quality of service is given by

$$
\phi(\sigma)=\phi^{n a}(\sigma)=\min \left\{\alpha \sigma+\left(1-\phi_{I}\right)\left(e^{(2 \lambda \sigma) / 3}-1\right) / 2, \bar{\phi}\right\}
$$

\footnotetext{
${ }^{27}$ To understand how the game would be affected if firms were making dynamic quantity choices, first assume that the entrant is myopic, but that the incumbent is not. Since the incumbent takes the entrant's current quantity as given when its sets its own quantity and the learning-by-doing process is only affected by firm E's current production, the incumbent cannot influence the entrant's process of experience acquisition through its quantity decision. Therefore, the two firms produce the short-run equilibrium quantities as if they were both myopic. Second, assume that the incumbent is myopic, but that the entrant is not. The entrant then internalizes the experience acquisition process in its quantity decision, and we have a learning-by-doing effect: the entrant produces a higher quantity, and since quantities are strategic substitutes, the incumbent produces less than in the short-run equilibrium.

${ }^{28}$ To extent that the incumbent prefers service-based competition over facility-based competition (because it earns wholesale profits under the former form of competition), it would have an incentive to slow down the entrant's acquisition of experience to retard facility-based entry.
} 
That is, the entrant's quality of service increases as it spends time in the market, until it reaches the threshold level, $\bar{\phi}$.

We now consider the case where access is a possibility. To simplify the analysis, we assume that the entrant reaches its maximum level of experience in the phase of facility-based competition. ${ }^{29}$ If service-based competition starts at date 0 and facility-based entry occurs at date $T$, at date $x$ the entrant's quality of service is

$$
\phi(\sigma)=\phi^{a}(x, T, r)= \begin{cases}\phi_{S}^{a}(x, r) & \text { if } \quad 0 \leq x \leq T \\ \phi_{F}^{a}(x, T, r) & \text { if } \quad x>T\end{cases}
$$

where

$$
\phi_{S}^{a}(x, r)=\alpha x+\left(1-\phi_{I}-2 r\right)\left(e^{2 \lambda x / 3}-1\right) / 2
$$

and $^{30}$

$$
\phi_{F}^{a}(x, T, r)=\min \left\{\alpha x+\left(1-\phi_{I}\right)\left(e^{2 \lambda x / 3}-1\right) / 2-r\left(e^{2 \lambda x / 3}-e^{2 \lambda(x-T) / 3}\right), \bar{\phi}\right\}
$$

As $\phi^{a}(x, T, r)$ decreases with $r$, a low access price allows the entrant to acquire market experience more rapidly. Moreover, if $r>0$, the entrant acquires experience at a slower pace under servicebased competition than under facility-based competition, because its customer base is smaller under the former form of competition than under the latter.

\subsection{The entrant's investment decision}

When there is no phase of service-based competition, the entrant's optimal investment date is given by Equation (3), with $\phi(\sigma)=\phi^{n a}(\sigma)$, where $\phi^{n a}(\sigma)$ is given by Equation (10).

When access is a possibility, the entrant requests access to the incumbent's infrastructure prior to building its infrastructure, as leasing access is a dominant strategy for the entrant under Assumptions 2(ii) and 3(ii). Besides, as $\phi_{F}^{a}$ increases with $x$ and goes to infinity when $x$ goes to infinity, there exists a date $\bar{x}(T, r)$ such that $\phi_{F}^{a}(x, T, r)=\bar{\phi}$ if and only if $x \geq \bar{x}(T, r) .{ }^{31}$ Therefore, the

\footnotetext{
${ }^{29}$ This is done without loss of generality.

${ }^{30}$ To determine $\phi_{F}^{a}(x, T, r)$, we use the fact that $\phi^{a}(x, T, r)$ is continuous at $x=T$.

${ }^{31}$ Note that $\bar{x}(T, r) \geq T$, as $\phi_{F}^{a}(x, T, r)$ is defined for $x \geq T$. To simplify the exposition, we drop the arguments of $\bar{x}$ in the rest of the text.
} 
entrant's discounted profit can be written as

$$
\Pi_{E}^{a}(T)=\int_{0}^{T} e^{-\rho x} \pi_{E}^{S}\left(\phi_{S}^{a}(x, r), r\right) d x+\int_{T}^{\bar{x}} e^{-\rho x} \pi_{E}^{F}\left(\phi_{F}^{a}(x, T, r)\right) d x+\int_{\bar{x}}^{\infty} e^{-\rho x} \pi_{E}^{F}(\bar{\phi}) d x-C(T) .
$$

This profit reads as follows. From date 0 to date $T$, the entrant requests access to the incumbent's infrastructure and accumulates market experience according to the process $\phi_{S}^{a}$. From date $T$ to date $\bar{x}$, there is facility-based competition, and the entrant continues accumulating experience, but at a faster pace, given by $\phi_{F}^{a}$. Finally, from date $\bar{x}$ on, the entrant has attained its maximum level of experience, and competes on the basis of facilities.

Assuming that $\Pi_{E}^{a}(T)$ is concave in $T,{ }^{32}$ the optimal facility-based entry date, $T^{a}$, satisfies the first-order condition,

$$
\begin{gathered}
-\left[\pi_{E}^{F}\left(\phi^{a}\left(T^{a}, T^{a}, r\right)\right)-\pi_{E}^{S}\left(\phi^{a}\left(T^{a}, T^{a}, r\right), r\right)\right] e^{-\rho T^{a}} \\
+\underbrace{\int_{T^{a}}^{\bar{x}} e^{-\rho x} \frac{d}{d T} \pi_{E}^{F}\left(\phi_{F}^{a}\left(x, T^{a}, r\right)\right) d x}_{A}-C^{\prime}\left(T^{a}\right)=0,
\end{gathered}
$$

since $e^{-\rho \bar{x}} \frac{\partial \bar{x}}{\partial T}\left[\pi_{E}^{F}\left(\phi_{F}^{a}\left(\bar{x}, T^{a}, r\right)\right)-\pi_{E}^{F}\left(\phi_{I}\right)\right]=0$, as $\pi_{E}^{F}\left(\phi_{F}^{a}\left(\bar{x}, T^{a}, r\right)\right)=\pi_{E}^{F}\left(\phi_{I}\right)$.

Compared to the first-order condition in our baseline model, given by (5), the first-order condition (11) contains an additional term that we denote by $A$. We find that $A$ is negative, as we have $\frac{d}{d T} \pi_{E}^{F}\left(\phi_{F}^{a}\left(x, T^{a}, r\right)\right)=\left(\partial \pi_{E}^{F} / \partial \phi\right) \times\left(\partial \phi_{F}^{a} / \partial T\right)$, and $\partial \pi_{E}^{F} / \partial \phi \geq 0$ and $\partial \phi_{F}^{a} / \partial T \leq 0$ for $x \geq T$. Therefore, term $A$ represents an incentive to enter on the basis of facilities at an early date. The intuition is that the quantity produced during a given period is smaller under service-based competition than under facility-based competition, as the entrant has to pay the access price under service-based competition. Therefore, quality improvements go faster under facility-based competition than under service-based competition. It follows that if the entrant delays its facility-based entry for a period $d T$, its quality improvements will be lower during this period, which affects all its future profit flows.

Since the first-order condition (11) is different from its equivalent in the baseline model, we reformulate the stepping stone effect. As in Section 3, the first-order condition with access can be written as

$$
F O C^{a}(T)=F O C^{n a}(T)+\pi_{E}^{S}\left(\phi^{a}(T, T, r), r\right) e^{-\rho T}+\widetilde{\Sigma}(T),
$$

\footnotetext{
${ }^{32}$ This is true if $C(\cdot)$ is sufficiently convex.
} 
where

$$
\begin{aligned}
\widetilde{\Sigma}(T, r)= & {\left[\pi_{E}^{F}\left(\phi^{n a}(0)\right)-\pi_{E}^{F}\left(\phi^{a}(T, T, r)\right)\right] e^{-\rho T}-\int_{T}^{\bar{\sigma}+T} e^{-\rho x} \frac{d}{d T} \pi_{E}^{F}\left(\phi^{n a}(x-T)\right) d x } \\
& +\int_{T}^{\bar{x}} e^{-\rho x} \frac{d}{d T} \pi_{E}^{F}\left(\phi_{F}^{a}(x, T, r)\right) d x
\end{aligned}
$$

and where $\bar{\sigma}$ is defined as the solution of $\phi^{n a}(\sigma)=\bar{\phi}$, with $\bar{\sigma}+T>\bar{x}$.

The first two terms in (12) are similar to the two terms in Equation (7) of the baseline model. Since $\phi^{a}(T, T, r)>\phi^{n a}(0)$ and $\pi_{E}^{F}(\phi)$ increases with $\phi$, the first term is negative, which tends to accelerate facility-based entry. It represents the cost of staying under service-based competition and not capturing the gains associated with experience acquisition. The second term corresponds to the benefit of remaining under service-based competition in terms of experience acquisition, and it gives an incentive to invest later than in the benchmark. Finally, the last term in Expression (12) was absent in the baseline model. This is the same term as in the first-order condition (11). As we have explained above, this term is negative, and it tends to accelerate facility-based entry. The idea is that because the entrant acquires market experience more rapidly under facility-based competition than under service-based competition, it now faces an opportunity cost of staying under service-based competition in terms of (lower) market experience.

\subsection{The relation between the access price and the date of facility-based entry}

In contrast to our baseline model, an increase of the access price has an ambiguous effect on the equilibrium date of facility-based entry with access, which is defined by the first-order condition (11). First, the access price has a direct effect on the investment date. If the level of experience $\phi^{a}$ is given and the access price $r$ increases, the replacement effect is reduced, as the entrant makes lower profits under service-based competition. This direct effect was present in our baseline model, and it tends to accelerate facility-based entry. Second, the access price has two indirect effects which go through the entrant's level of experience, and were absent in the baseline model. If the access price increases, the entrant's acquisition of experience slows down under service-based competition, because the quantity produced by the entrant decreases. The entrant's experience at a given date, $\phi^{a}$, therefore decreases, which in turn affects the first two terms of Equation (11): $(i)$ the entrant's profit incentive, $\Delta \pi_{E}$, decreases, which tends to delay facility-based entry; ${ }^{33}$ (ii) term $A$, which

\footnotetext{
${ }^{33}$ Note that with another competition setting, this effect will also be present, but that it might go in the opposite direction, as $\Delta \pi_{E}$ does not necessarily increase with $\phi^{a}$. For example, this is not true in the Hotelling setting that
} 
represents the loss in experience acquisition associated with waiting, can either increase or decrease. Indeed, for $\phi^{a}\left(x, T^{a}, r\right)<\phi_{I}$, we have

$$
\frac{\partial}{\partial r}\left[\frac{\partial \pi_{E}^{F}}{\partial \phi} \frac{\partial \phi^{a}}{\partial T}\left(x, T^{a}, r\right)\right]=-\frac{8}{27} e^{2(x-T) \lambda / 3} \lambda\left[\left(1-\phi_{I}+2 \phi^{a}\left(x, T^{a}, r\right)\right)+2 r \frac{\partial \phi^{a}}{\partial r}\left(x, T^{a}, r\right)\right] .
$$

Since $\left(1-\phi_{I}+2 \phi^{a}\left(x, T^{a}, r\right)\right)>0$ and $\partial \phi^{a} / \partial r<0$, this expression can be either positive or negative. Therefore, term $A$ can either increase or decrease with $r$.

Interestingly enough, and in contrast to our baseline model, the stepping stone effect now depends on the access price, $r$. Using Equation (7) we find that

$$
\begin{aligned}
\frac{\partial \widetilde{\Sigma}(T, r)}{\partial r}= & -\frac{\partial \pi_{E}^{F}\left(\phi^{a}(T, T, r)\right)}{\partial \phi} \frac{\partial \phi^{a}(T, T, r)}{\partial r} e^{-\rho T}+\int_{T}^{\bar{x}} e^{-\rho x} \frac{\partial}{\partial r} \frac{d}{d T} \pi_{E}^{F}\left(\phi^{a}(x, T, r)\right) d x \\
& +\frac{\partial \bar{x}}{\partial r} \frac{d}{d T} \pi_{E}^{F}\left(\phi_{F}^{a}(\bar{x}(T, r), T, r)\right) e^{-\rho \bar{x}}
\end{aligned}
$$

The first term is positive as $\partial \pi_{E}^{F} / \partial \phi>0$ and $\partial \phi^{a} / \partial r<0$, whereas the second and third terms can be either positive or negative. Though we cannot determine the sign of $\partial \widetilde{\Sigma}(T, r) / \partial r$ analytically, simulations suggest that when the access price $r$ increases, the value of the stepping stone effect is reduced for low values of $T$ and increased for high values of $T$.

Therefore, if the entrant's optimal date of facility-based entry is early, increasing $r$ will reduce both the replacement effect and the stepping stone effect, which will in turn lead to an earlier investment date. However, if the date of facility-based entry is late, increasing the access price softens the replacement effect but increases the value of the stepping stone effect. In this case, a higher access price might delay facility-based entry. We can then state the following Proposition.

Proposition 3 When the acquisition of market experience depends on the entrant's customer base, increasing the access price tends to accelerate the entrant's investment, compared to the benchmark, if facility-based entry is a short-term possibility, and to delay investment if facility-based entry is a long-term possibility.

If facility-based entry is a short-term possibility, we obtain the same result than in the baseline model: increasing the access price accelerates investment. The regulator therefore faces the standard trade-off between static efficiency and investment incentives, which it may solve with a more sophisticated access price regulation. If facility-based entry is only a long-term possibility,

we developed in our working paper version, Bourreau and Drouard (2010). 
however, we obtain a different result. In this case, due to the learning-by-doing effect, the regulator can achieve both static efficiency and an early investment by setting a low access price. In other words, service-based competition can facilitate facility-based entry per se, without the need to implement a fine-tuned regulation.

As an illustration, we propose a numerical example with the following parameter values, $\phi_{I}=$ $\bar{\phi}=0.75, \rho=0.05, \alpha=0$ and $r=0.1,{ }^{34}$ and determine the overall impact of an increase of the access price, $r$, when there is a phase of service-based competition. We define $R E(T, r)=$ $\pi_{E}^{S}\left(\phi^{a}(T, T, r), r\right) e^{-\rho T}$, which represents the replacement effect. Note that $\partial R E / \partial r<0$; that is, a higher access price softens the replacement effect. Figure 1 shows the impact of a marginal increase of the access price on the date of facility-based entry, as a function of the strength of the learning-by-doing effect $\lambda$ (on the horizontal axis) and the date of facility-based entry with access $T^{a}$ (on the vertical axis).

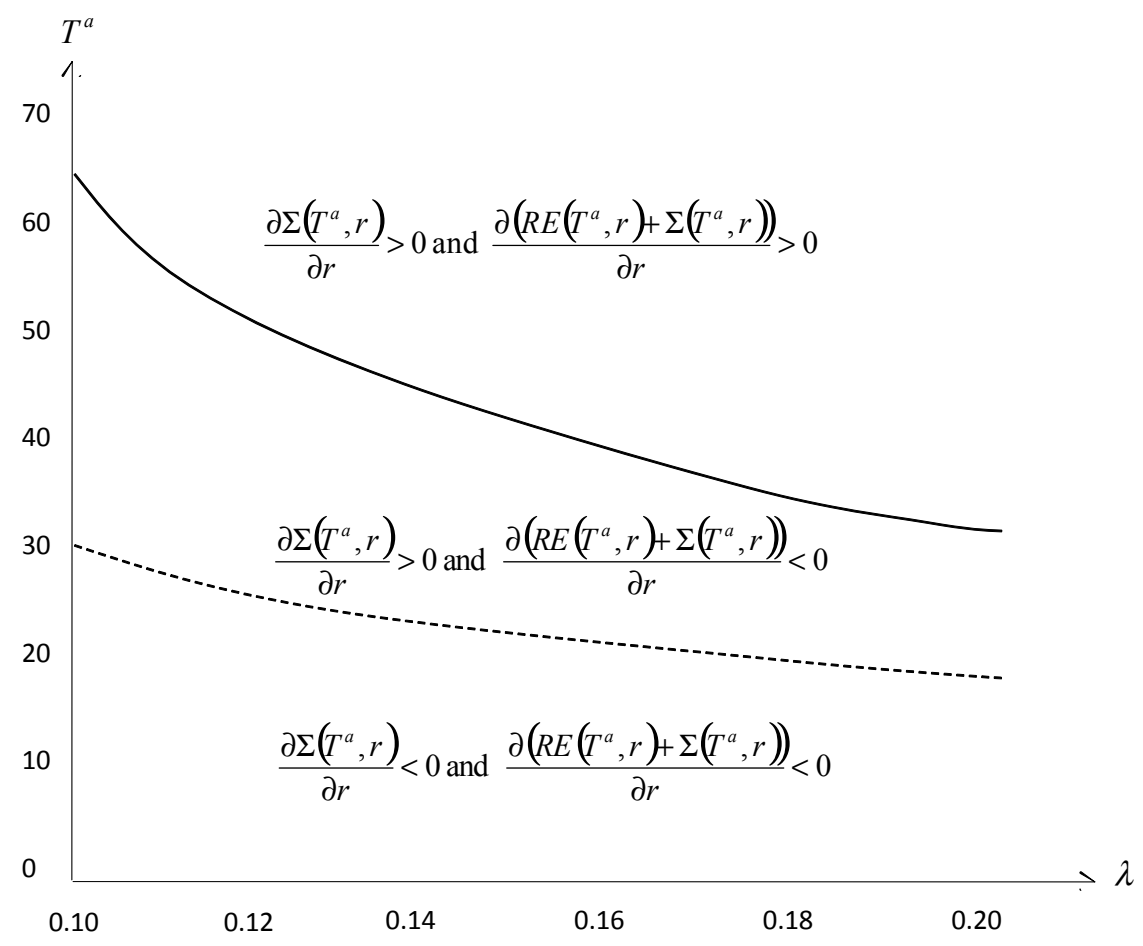

Figure 1: Impact of a marginal increase of the access price on the replacement effect and the stepping stone effect.

There are three zones in Figure 1. First, if the date of facility-based entry is below the dotted line, an increase of the access price decreases the value of the stepping stone effect. Second, if the

\footnotetext{
${ }^{34}$ Note that this value of $r$ does not necessarily correspond to the socially optimal $r$.
} 
date of facility-based entry is above the dotted line and below the thick line, an increase of the access price increases the value of the stepping stone effect, but is not large enough to compensate for the decrease of the replacement effect. In these two zones, therefore, increasing the access price accelerates facility-based entry, which corresponds to the standard result. Third, if the date of facility-based entry is above the thick line, an increase of the access price increases the stepping stone effect, and the increase of the stepping stone effect dominates the decrease of the replacement effect. Therefore, if the date of facility-based entry with access is sufficiently high, an increase of the access price delays facility-based entry, in contrast with our baseline model.

Finally, note that the replacement effect and the stepping stone effect vary with the learningby-doing parameter $\lambda$. Since the entrant accumulates experience at a faster pace when $\lambda$ increases, the replacement effect increases with $\lambda$, which tends to delay the date of facility-based entry. The effect of the learning-by-doing parameter on the stepping stone effect is less clear-cut. On the one hand, an increase in $\lambda$ raises the benefits for the entrant from prolonging the phase of service-based competition, in terms of higher profits under facility-based competition. On the other hand, the opportunity cost in terms of lower market experience accumulation under service-based competition also increases $\lambda$. Simulations suggest that overall, the stepping stone effect decreases (resp., increases) with $\lambda$ if facility-based entry is a long-term (resp., short term) possibility. Therefore, when the date of facility-based entry without access is early, an increase in the learning-by-doing parameter reinforces both the replacement effect and the stepping stone effect, and delays the date of facility-based entry with access.

\section{Conclusion}

In this paper, we have studied how a phase of service-based competition, where an entrant leases access to an incumbent's infrastructure, affects the entrant's incentives to invest in an alternative infrastructure, when it acquires market experience progressively. We have shown that the phase of service-based competition has two effects on the date of facility-based entry: a replacement effect, and a "stepping stone" effect, which is due to the entrant's acquisition of experience.

When experience acquisition depends only on the time spent in the market and facility-based entry is a long-term possibility, the stepping stone effect increases the entrant's investment incentives, and thus accelerates facility-based entry. However, if facility-based entry is possible in the short term (due to low investment costs, for example), the stepping stone effect delays the 
entrant's investment. The intuition is that the entrant has then incentives to prolong the phase of service-based competition to acquire more experience. We have then shown that a phase of service-based competition delays facility-based entry if prospects of facility-based competition are sufficiently high, and that it can accelerate facility-based entry if prospects are very weak and experience acquisition has a strong effect on the entrant's profits.

We have also analyzed the case where experience acquisition depends on the entrant's customer base. If facility-based entry is a short-term possibility, we obtain the same result as in our main model: increasing the access price enhances investment incentives, but at the cost of lower static efficiency. However, if facility-based entry is viable only in the long run, a low access price, which is pro-competitive, also allows the entrant to acquire experience rapidly, and thus accelerates facilitybased entry.

From a policy perspective, our results suggest that regulators face a trade-off between servicebased and facility-based competition when facility-based entry is possible in the short term. Even though entrants can acquire experience during the phase of service-based competition, facility-based entry is delayed. A fine-tuned regulation of the access price (e.g., an access price increasing over time) is therefore warranted. By contrast, if facility-based entry appears to be viable only in the long run, a phase of service-based competition with a low access price can effectively serve as a stepping stone for a new entrant.

\section{References}

Arrow, K., 1962. Economic Welfare and the Allocation of Resources for Invention. In NBER Conference, "The Rate and Direction of Inventive Activity: Economic and Social Factors," Princeton University Press, Princeton.

Avenali, A., Matteucci, G. and Reverberi, P., 2010. Dynamic access pricing and investment in alternative infrastructures. International Journal of Industrial Organization, 28(2), 167-175.

Bacache, M., Bourreau, M. and Gaudin, G., 2013. Dynamic Entry and Investment in New Infrastructures: Empirical Evidence from the Fixed Broadband Industry. Review of Industrial Organization, forthcoming.

Bourreau, M. and Doğan, P., 2005. Unbundling the local loop. European Economic Review, 49, 173-199. 
Bourreau, M. and Doğan, P., 2006. "Build-or-Buy" Strategies in the Local Loop. American Economic Review, 96(2), 72-76.

Bourreau, M., Doğan, P. and Manant, M., 2010. A critical review of the ladder of investment approach. Telecommunications Policy, 34(11), 683-696.

Bourreau, M. and Drouard, J., 2010. Stepping stone or stonewall? Progressive entry and the incentives to invest in alternative infrastructures. Telecom ParisTech Working Paper No. ESS-1017.

Cambini, C. and Jiang, Y., 2009. Broadband investment and regulation: A literature review. Telecommunications Policy, 33(10-11), 559-574.

Cave, M., 2006. Encouraging infrastructure competition via the ladder of investment. Telecommunications Policy, 30, 223-237.

Crandall, R.W. and Sidak, J.G., 2007. Is Mandatory Unbundling the Key to Increasing Broadband Penetration in Mexico? A Survey of International Evidence. Mimeo. http://ssrn.com/abstract $=996065$.

Distaso, W., Lupi, P. and Manenti, G. M., 2009. Static and Dynamic Efficiency in the European Telecommunications Market: The Incentives to Invest and the Ladder of Investment. In I. Lee, ed., Handbook of Research on Telecommunications Planning and Management, Vol. 1, Chap. 1, pp. 1-14. Hershey: Information Science Reference.

EU, 2010. Commission recommendation of 20 September 2010 on regulated access to next generation access networks (NGA). Retrieved from <http://eurlex.europa.eu/LexUriServ/LexUriServ.do?uri=OJ:L:2010:251:0035:0048:EN:PDF>.

Foros, Ø., 2004. Strategic Investments with Spillovers, Vertical Integration and Foreclosure in the Broadband Access Market. International Journal of Industrial Organization, 22, 1-24.

Gallini, N., 1984. Deterrence by Market Sharing: A Strategic Incentive for Licensing. American Economic Review, 74(5), 931-941.

Gans, J., 2001. Regulating Private Infrastructure Investment: Optimal Pricing for Access to Essential Facilities. Journal of Regulatory Economics, 20(2), 167-189. 
Gans, J., 2007. Access Pricing and Infrastructure Investment. In Dewenter, R. and Haucap, J. (eds.), Access Pricing: Theory and Practice, Elsevier Science, Amsterdam, Chapter 2.

Guthrie, G., 2006. Regulating Infrastructure: The Impact on Risk and Investment. Journal of Economic Literature, 44, 925-972.

Hausman, J.A. and Sidak, J.G., 2005. Did Mandatory Unbundling Achieve its Purpose? Empirical Evidence from Five Countries. Journal of Competition Law and Economics, 1(1), 173-245.

Hazlett, T. and Bazelon, C., 2005. Regulated Unbundling of Telecommunications Networks: A Stepping Stone to Facilities-Based Competition? George Mason University. Available at: http://mason.gmu.edu/ thazlett/pubs/Stepping\%20Stone\%20TPRC.10.04.05\%20.pdf.

Hori, K. and Mizuno, K., 2006. Access pricing and investment with stochastically growing demand. International Journal of Industrial Organization, 24(4), 795-808.

Katz, M.L. and Shapiro, C., 1985. Network Externalities, Competition, and Compatibility. American Economic Review, 75, 424-440.

Nitsche, R., and Wiethaus, L., 2011. Access Regulation and Investment in Next Generation Networks - A Ranking of Regulatory Regimes. International Journal of Industrial Organization, 29(2), 263-272.

Schutz, N. and Trégouët, T., 2008. The Ladder of Investment in Telecoms: Stairway to Heaven or Highway to Hell? Unpublished manuscript.

Valletti, T., 2003. The theory of access pricing and its linkage with investment incentives. Telecommunications Policy, 27, 659-675.

Vareda, J., 2010. Access regulation under asymmetric information about the entrant's efficiency. Information Economics and Policy, 22(2), 192-199.

Vareda, J. and Hoernig, S., 2010. Racing for Investment under Mandatory Access. The B.E. Journal of Economic Analysis $\&$ Policy, 10(1), Article 67. 


\section{Appendix}

\section{Appendix A: Second-order condition without access}

Using (4), we find that

$$
\frac{d^{2} \Pi_{E}^{n a}}{d T^{2}}=\rho^{2} e^{-\rho T} \int_{0}^{\infty} e^{-\rho x} \pi_{E}^{F}(\phi(x)) d x-C^{\prime \prime}(T)
$$

The firm term is positive or negative. Therefore, $\Pi_{E}^{n a}$ is concave if $C(T)$ is sufficiently convex.

\section{Appendix B: Second-order condition with access}

Using (5), we find that

$\frac{d^{2} \Pi_{E}^{a}}{d T^{2}}=\rho e^{-\rho T}\left[\pi_{E}^{F}(\phi(T))-\pi_{E}^{S}(\phi(T), r)\right]-e^{-\rho T} \phi^{\prime}(T)\left[\frac{\partial \pi_{E}^{F}}{\partial \phi}(\phi(T))-\frac{\partial \pi_{E}^{S}}{\partial \phi}(\phi(T), r)\right]-C^{\prime \prime}(T)$.

The firm term is positive under Assumption 2(ii), whereas the second term can be either positive or negative. Therefore, $\Pi_{E}^{a}$ is concave if $C(T)$ is sufficiently convex. 